\title{
BMJ Open Improving social inclusion for people with dementia and carers through sharing dance: a qualitative sequential continuum of care pilot study protocol
}

Mark W Skinner, ${ }^{1}$ Rachel V Herron, ${ }^{2}$ Rachel J Bar, ${ }^{3}$ Pia Kontos, ${ }^{4}$ Verena Menec ${ }^{5}$

To cite: Skinner MW, Herron RV, Bar RJ, et al. Improving social inclusion for people with dementia and carers through sharing dance: a qualitative sequential continuum of care pilot study protocol. BMJ Open 2018;8:e026912. doi:10.1136/ bmjopen-2018-026912

- Prepublication history for this paper is available online. To view these files, please visit the journal online (http://dx.doi org/10.1136/bmjopen-2018026912).

Received 27 September 2018 Revised 10 0ctober 2018 Accepted 11 October 2018

A Check for updates

(C) Author(s) (or their employer(s)) 2018. Re-use permitted under CC BY-NC. No commercial re-use. See rights and permissions. Published by BMJ.

${ }^{1}$ Trent School of the

Environment, Trent University, Peterborough, Ontario, Canada ${ }^{2}$ Department of Geography, Brandon University, Brandon, Manitoba, Canada

${ }^{3}$ Canada's National Ballet School and Department of Psychology, Ryerson University, Toronto, Ontario, Canada

${ }^{4}$ Toronto Rehabilitation Institute - University Health Network, Toronto, Ontario, Canada

${ }^{5}$ Community Health Sciences, University of Manitoba, Winnipeg, Winnipeg, Canada

Correspondence to

Dr Mark W Skinner;

markskinner@trentu.ca

\section{ABSTRACT}

Introduction This study examines the potential of dance to improve social inclusion for people living with dementia and carers. Research suggests that arts-based programmes can improve the health of people living with dementia and carers; however, little is known about how these programmes might address barriers to social inclusion. Addressing barriers requires the development and evaluation of accessible, non-stigmatising and affordable programmes that facilitate social inclusion across the continuum of institutional, community and household care settings.

Methods and analysis The study involves a qualitative sequential pilot study of the innovative Baycrest NBS Sharing Dance Seniors programme underway in non-metropolitan regions of two Canadian provinces. It focuses on the remotely instructed delivery of the programme in care facilities, community centres and households. The study involves five phases of observations, diaries, focus groups and interviews with programme participants (people living with dementia), carers, coordinators, instructors and volunteers as well as critical reflections among research investigators and knowledge users. NVivo-based thematic and narrative analyses of the qualitative data will produce new knowledge about the experiences, effectiveness and challenges of the dance programme that will inform understanding of whether and in what ways it increases social inclusion and quality of life for older people living with dementia and carers. The findings will identify opportunities for programme expansion and support the further development of arts-based approaches.

Ethics and dissemination The study is approved by the Research Ethics Boards at Trent University and Brandon University, and by participating organisations according to their governance procedures. The perspectives of people living with dementia and carers are incorporated throughout the study (from design to dissemination) and the study adheres to the ethical considerations when including people with dementia. A series of publicly available reports, seminars and symposia will be undertaken in collaboration with knowledge user and collaborating organisation partners.

\section{INTRODUCTION}

Dementia, inclusive of a range of progressive neurodegenerative conditions such as Alzheimer's disease, affects approximately

\section{Strengths and limitations of this study}

- The study is one of the first to examine the potential of arts-based programmes for improving social inclusion across the continuum of institutional, community and household care settings.

- The study draws together the perspectives of people living with dementia, carers, staff and volunteers, with the expertise of other knowledge users and community partners, in collaboration with expertise from dementia care, social gerontology and artsbased health researchers.

- The study will help develop further understanding of the experiences, effectiveness and challenges of implementing promising dance programmes to increase social inclusion and quality of life for older people living with dementia and their carers.

- A limitation is that this is a pilot study with an exploratory qualitative design and as such cannot provide quantitative outcome measures of the programme under investigation.

46.8 million people worldwide. ${ }^{1}$ Over the course of the condition, people living with dementia and their carers often face barriers to meaningful participation and social inclusion. ${ }^{23}$ Social inclusion is a complex, multidimensional process and outcome that results from supporting the rights for full participation with resources and services in relationships and activities across economic, social and cultural spheres. ${ }^{4}$ Addressing these issues requires careful attention to the diverse experiences and contexts of people living with dementia and their carers ${ }^{5}$ as well as developing accessible, non-stigmatising and affordable programmes that facilitate social inclusion across the continuum of institutional, community and household care settings. ${ }^{6}$ Arts-based health programmes such as painting, music and dance have been identified as effective in improving the physical, emotional and neurological health and well-being of people living with dementia, as 
well as providing opportunities for them and their carers to develop stronger social support networks. ${ }^{7}$ What is missing is research into the experiences, effectiveness and scalability of arts-based programmes for social inclusion of people living with dementia and their carers.

This study examines the potential for dance-based programmes to improve social inclusion among people living with dementia and their carers. The focus on dance is important because it can offer people living with dementia opportunities to express their thoughts, memories and emotions, and relate with others. ${ }^{8}$ Dance programmes support the expressive capabilities of people living with dementia, which frequently are undermined by discourses that focus on the cognitive decline associated with dementia and fail to consider the body's power of expression. ${ }^{9}$ In addition, depending on the mode of dance, it can provide opportunities for physical touch and connection. As such, dance programmes are particularly well-suited to providing opportunities for people living with dementia to connect with others in spite of the cognitive changes and social challenges associated with degenerative conditions. ${ }^{10}$ To date, however, research on social inclusion as an outcome of arts-based programmes more broadly has been relatively limited, with little attention to the processes involved in facilitating social inclusion through the arts, and even less attention directed to the social inclusion benefits of dance more specifically. ${ }^{11}$

To address this gap, we are examining the implementation of an innovative, community-oriented, dance programme called Baycrest NBS Sharing Dance Seniors, which is a joint venture between Canada's National Ballet School (NBS) and Baycrest Health Sciences. [Canada's National Ballet School (NBS) is a Toronto-based internationally renowned ballet school best known for training elite dancers and for its innovative Sharing Dance programmes. Baycrest Health Sciences, also located in Toronto, is a residential long-term care facility and leading centre for teaching and research on ageing in Canada. Both organisations have collaborated in developing Baycrest NBS Sharing Dance Seniors since 2014.] The Sharing Dance Seniors programme is unique in Canada and internationally in its aim to make dance accessible to older adults with a range of physical and cognitive abilities, including people living with dementia. ${ }^{12}$ The programme involves a suite of remotely instructed (streamed) dance sessions to participants in weekly facilitator-led dance sessions in institutional, community and/or household settings with on-site facilitators supporting participants. The programme is currently being piloted for nationwide expansion as part of a multisectoral strategic partnership with the Public Health Agency of Canada ${ }^{13}$ in collaboration with long-term (residential) care homes, regional home care providers and community support agencies. The research protocol introduced in this paper focuses on sequential multiphase pilot studies of the Baycrest NBS Sharing Dance Seniors programme underway in two non-metropolitan regional centres in the Canadian provinces of Ontario and Manitoba. The pilot studies expand the prevailing biomedical and public health foci on the neurological and therapeutic outcomes of the dance programme (eg, falls prevention) by emphasising the ways in which it supports participants' and carers' participation, self-expression and social inclusion.

Sharing Dance Seniors is offered at two levels since it is designed to be accessible to people with significant cognitive and/or physical impairment such as dementia (level 1 ), and to be accessible to people with minor impairments associated with ageing (level 2). Level 1 is danced in $45 \mathrm{~min}$ sessions from a seated position to reduce the risk of falling, and level 2 includes both seated and standing options for participants to decide for themselves what feels most appropriate. Level 2 is danced over the course of an hour. Both levels follow a standardised (replicable) programme that outlines a series of dances (eg, warm-up, dances of upper and lower extremities, narrative dances, mirroring and improvisation and singing), each with specific creative and physical goals and rationale. The programme moves beyond dance-as-therapy, which is the focus of many dance programmes, ${ }^{11}$ by emphasising the importance of creative self-expression as itself an objective of the programme. For example, opportunities are provided for participants to express their life-stories through movement, which is a key goal of the narrative dance approach. Sharing Dance Seniors aims to provide participants with a dance experience as similar as possible to that of any traditional dance class, while simultaneously prioritising accessibility to older adults with physical and/or cognitive challenges. These objectives are distinct from dance therapy, which aims to improve neuropsychiatric symptoms such as agitation and cognitive and physical functioning, largely overlooking what are considered 'softer' positive benefits including empowerment, communication and creative self-expression, communal spirit, pleasurable experience and sociability. ${ }^{14}$ The aims of the dance programme align directly with this study's interest in social inclusion as they prioritise expressive capacities and social interaction in the group rather than positioning these processes and outcomes as secondary to physical and cognitive outcomes.

\section{ARTS-BASED HEALTH RESEARCH}

Research on arts-based health programmes has focused predominantly on measuring outcomes of the programmes: preventing falling, improving balance, mood, cognition and physical functioning, and decreasing problematic behaviours. ${ }^{15-17}$ There are studies that have documented improvements in social interactions between care workers and people living with dementia including enhanced communication and enjoyment for both. ${ }^{17}{ }^{18}$ There is also evidence that arts-based programmes can enliven the individual living with dementia, which studies have linked to improvements in person-centred care and relational citizenship. ${ }^{89}$ Indeed, Dupuis $e t a l^{19}$ argue that arts-based approaches can create transformative spaces that challenge dominant ideas about dementia, 
and support the development of new forms of mutual support, caring and relating between persons living with dementia and others.

With these emergent findings in mind, this study will address several important gaps in scholarship. First, there is a need for research that includes a focus on both outcomes and processes, particularly as they relate to the dynamics of social inclusion. Unlike some of the other outcomes measured in previous arts-based studies (eg, functional balance), social inclusion is a dynamic process rather than a single outcome. Dedicated research is needed to examine how arts-based approaches can facilitate social inclusion for people with dementia and carers as well as what contextual factors influence the success or failure of art-based programming in achieving this objective. Furthermore, much of the literature has relied on data from care staff and family carers in the evaluation of the impact of arts-based programmes on both carers and people with dementia, rather than focusing on the experiences of people with dementia themselves. More specifically, there is very little research on arts-based programmes that includes people in the earlier stages of dementia who are living at home. The majority of artsbased programmes have taken place in institutional care settings, such as long-term care facilities and residential care homes, with people in the later stages of dementia. ${ }^{11}$ While a smaller number of studies in the last few years have focused on community settings with people living with mild-to-moderate dementia, the diverse capacities of individuals to engage meaningfully in arts-based programmes have not been adequately explored. ${ }^{20}$ Finally, research has paid limited attention to how people experience and express meaning and inclusion through the $\operatorname{arts}^{11}$; a multidimensional approach to conceptualising and operationalising social inclusion is needed. This study addresses these gaps by examining an arts-based (dance) programme across the continuum institutional, community and household care settings in non-metropolitan (regional and rural) locales, and by framing the analysis within a multidimensional conceptualisation of age-related social inclusion.

Our definition of social inclusion as a multidimensional process and outcome draws on a conceptual framework developed at the Irish Centre for Social Gerontology. Recognising the often-unidimensional limitations of existing models, Walsh et at incorporated into their framework the diversity of and interlinkages among contextual and individual elements of social inclusion. Their framework provides a multidimensional and multilayered approach to understanding factors influencing exclusion and inclusion, specifically social relations, access to services, financial resources, transportation and mobility and safety and security, as well as the broader influence of macroeconomic forces, place and community, individual capacities and life-course trajectories. This study adopts and expands the framework by Walsh et $a l,{ }^{4}$ which has yet not been used to explore the experiences of people living with dementia or of carers. There is limited knowledge of how relationships of care, place and community capacity, and individual histories interact with other factors to influence social inclusion. There is also a lack of understanding how arts-based programmes can address these factors, individually and in combination.

\section{RESEARCH OBJECTIVES}

Against the backdrop of these issues and gaps in knowledge, the overall goal of the Improving Social Inclusion for People with Dementia and Carers through Sharing Dance study is to investigate the potential to improve social inclusion of people living with dementia and their carers through the expansion of the Baycrest NBS Sharing Dance Seniors programme. With an exploratory emphasis on building new knowledge across the continuum of institutional, community and household care settings, the research objectives are threefold: (1) to examine the experiences of older people living with dementia and their carers participating in the programme as they relate to the multiple dimensions of social inclusion; (2) to assess the effectiveness of the remote delivery of the programme to enhance social inclusion processes and outcomes and (3) to identify the challenges of expanding the programme (eg, programmatic and contextual factors that influence the success or failure of the programme) in terms of improving social inclusion. Underlying these objectives is the methodological aim to develop and pilot new qualitative approaches to researching arts-based programmes with people living with dementia.

\section{RESEARCH DESIGN AND METHODS}

The research involves two Sharing Dance Seniors pilot studies in Peterborough, Ontario (regional population of $165000,20 \%$ of which are aged over 65 years) and in Brandon, Manitoba (regional population of $180000,18 \%$ of which are aged over 65 years). ${ }^{21}{ }^{22}$ These regions are representative of Canada's ageing populations and associated prevalence of age-related degenerative diseases, which are most proportionate in non-metropolitan (rural) regions. ${ }^{23}$ The pilot study sites are located in comparable provincial jurisdictions and were selected based on the availability of local and regional partners interested in running the dance programme (eg, Alzheimer Societies, community support agencies and seniors centres) and proximity to the research investigators from Trent University and University of Toronto, and Brandon University and University of Manitoba, respectively. The pilot studies are designed to run sequentially, beginning with the Peterborough Pilot Study focusing on the delivery of the programme for older adults with minor impairments associated with ageing (ie, level 2). This will be followed by the Brandon Pilot Study focusing on the delivery of the programme for older adults with significant cognitive and/or physical impairments such as dementia (ie, level 1). As outlined in table 1, each pilot study begins with a single site dress rehearsal of the remotely streamed 


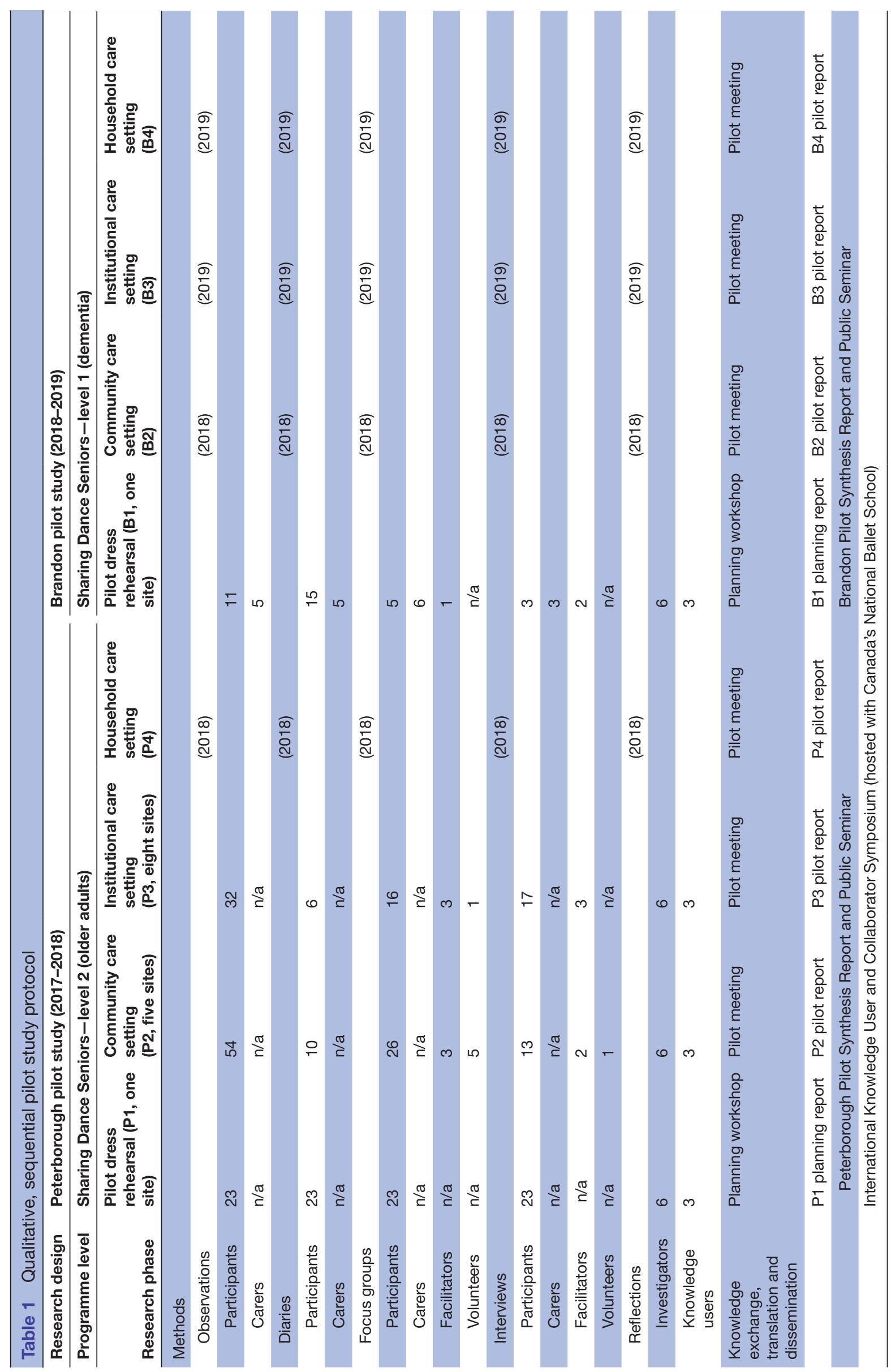


programme (referred to as $\mathrm{P} 1$ and $\mathrm{B} 1$ ), which facilitates a collaborative approach to developing participant recruitment and data collection strategies with local partners and knowledge users. The initial phase is followed by a broader expansion of data collection in multiple community sites (P2, B2), institutional settings (P3, B3) and individual households (P4, B4) (table 1 reports recruitment of research participants at the half-way point of the sequential pilot study phases). The Peterborough Pilot Study began in April 2017 and will be completed in June 2018, and the Brandon Pilot Study began in April 2018 and will be completed in June 2019. The iterative, sequential design of the pilot studies allows for the co-development, with the researchers, knowledge users and collaborators, of the partnerships, research instruments and approaches required to achieve the study objectives.

\section{PUBLIC PARTICIPANT INVOLVEMENT}

Underlying the study design is a commitment to ensuring meaningful engagement with people living with dementia and carers, as well as other knowledge user collaborators from the dementia and dance sectors (eg, Alzheimer Society of Ontario, Alzheimer Society of Manitoba; Canada's National Ballet School, Canada's Royal Winnipeg Ballet School). The perspectives of people living with dementia and carers are incorporated throughout the study (from design to dissemination) via a project advisory board including membership from Alzheimer Society client advisory committees (people living with dementia) and support groups (carers) in the pilot study regions. Following a community-based approach, each pilot study involves working closely with community partners in Peterborough and Brandon to recruit participants to the programme. The development of the exploratory study design also was collaborative with the Alzheimer Society client advisory committees and support groups and will continue to include the perspectives of people living with dementia and carers in every phase of the study.

\section{DATA COLLECTION}

The research design features five qualitative phases (observations, diaries, focus groups, interviews and reflections) to be carried out in each of the Peterborough and Brandon pilot studies. In terms of participant recruitment, no diagnostic tests (eg, Mini-Mental Health Examination) are performed to assess the cognitive capacity of participants with dementia. Nobody is excluded from participating in the study. All participants are recruited first to participate in the Sharing Dance Seniors programme and they are then provided with a letter of information and consent form to voluntarily participate in the study.

First, observations of participants in the programme are conducted in situ by the researchers to explore their lived experiences as they relate to processes and outcomes of social inclusion (objective 1). The observations include detailed descriptions of the actions, expressions and interactions of participants in the programme to inform our analysis of multiple dimensions of social inclusion, particularly those related to social relations, mobility and safety. For example, observations focus on the degree and nature of social interaction as well as safety (eg, comfort with dance moves, fellow participants and the facilitator).

Second, diaries with programme participants are used to explore the lived experiences of the programme as they relate to processes and outcomes of social inclusion (objective 1). People living with dementia and carers record their personal experiences throughout the programme in a weekly semi-structured journal using written, oral and/or video communication (paper booklets and iPads). Researchers assist people living with dementia with their diaries after the programme, as needed. Diary questions focus on elucidating elements of social inclusion within the participants' and carers' experiences (eg, changes in social connections over the course of the programme, barriers to participation due to transportation and mobility issues, financial issues and safety concerns) to enhance the other data collected regarding social inclusion in depth and with contextual sensitivity.

Third, focus groups with programme participants and carers as well as with programme coordinators, instructors and volunteers are moderated regarding the effectiveness of the programme delivery (objective 2) and the challenges of implementing the programme (objective 3). This allows for an examination of the breadth of experiential, programmatic and contextual factors that influence the effectiveness of the programme to improve social inclusion. The focus groups explore similarities and differences among the modes of delivery, care settings and geographic locales, and include discussion questions about broader contextual influences on social inclusion (eg, impact of changing economic circumstances and community dynamics such as volunteerism on access to services).

Fourth, interviews with programme participants and carers as well as with programme coordinators, instructors and volunteers will explore the effectiveness of the multimodal programme delivery and identify the challenges of implementing the programme (objectives 2 and 3 ). In-depth, $90 \mathrm{~min}$ audio-recorded interviews with a subset of programme participants, and all of the coordinators, instructors and volunteers will explore the success and/ or failure of the programme in relation to experiential, programmatic and contextual factors that influence the effectiveness of the programme to improve social inclusion. Semi-structured interview questions will explore in greater depth the key issues and connections among the multiple dimensions of social inclusion that emerge from the focus group discussions.

Fifth, critical reflections among the research investigators and knowledge users will serve to identify opportunities and promising practices for expanding the programme in various types of community and collaborative settings (objective 3). In-depth reflections regarding the challenges and opportunities experienced and/or 
observed by the research team (comprising the investigators, knowledge user collaborators from NBS and research personnel) are recorded as part of weekly 1 hour meetings to build a reflective dataset as the programme expands through the pilot phases (ie, P1 to P4, B1 to B4). A semi-structured guide is designed to elicit conceptual, methodological, empirical and applied issues for discussion and critical reflection.

\section{DATA ANALYSIS}

Sequential and iterative qualitative data analysis will take place during the multiphased data collection to support the refinement of research instruments as well as communication of preliminary findings with community partners. Management and analysis of all phases of the study will be supported by Nvivo V.11 software. Thematic content analysis of text-based observations as well as diaries will inform the focus group discussions of the effectiveness and challenges of the Sharing Dance Seniors programme. Building on the observational and diary phases, thematic analyses of focus group transcripts inform the in-depth inquiry of emergent social inclusion themes in interviews. Thematic analysis of the recorded researcher and knowledge user reflections will provide the foundation for narratives of promising practices relating to the development and implementation of the research design as well as the implementation and expansion of the dance programme. These analyses will inform a series of knowledge exchange reports and seminars for each phase of the pilot studies that will be disseminated to participants, community leaders and knowledge users (table 1).

\section{OUTCOMES}

The Improving Social Inclusion for People with Dementia and Carers through Sharing Dance study will enhance our understanding of how people living with dementia experience programmes within household, community and institutional settings. The majority of research on arts-based health programmes has focused on institutional settings; yet, the majority of the population living with dementia reside in the community and should have access to meaningful social activities no matter where they live geographically (ie, metropolitan, urban, rural or remote) ${ }^{24}$ Similar to Marshall $e t a l^{25}$ this study will address questions about how to provide such dementia-friendly activities, in this case by examining the effectiveness of video-streaming arts-based programmes with regard to social inclusion. It will also address important community development questions about the challenges of trying to expand programmes to different types of settings and the capacity of different organisations to support programme expansion.

Methodologically, the study confronts some of the challenges in dance programme research. A growing body of literature has captured the benefits of dance in older adulthood. ${ }^{26}$ One criticism that is described frequently in reviews of the field, however, is the general lack of specificity of the dance practices used. ${ }^{27}$ Without detailed descriptions of the dance programmes, even if a study captures positive outcomes, replicability is compromised, greatly limiting the utility of such research. Baycrest NBS Sharing Dance Seniors is based on a programme that identifies specific goals for each dance performed within a class. While the specific steps, music and context of a dance may vary, the programme is designed to achieve replicable goals by anyone trained to deliver the programme such as the institutional, community and household facilitators involved in the Peterborough and Brandon pilot studies. Findings from this study will therefore contribute to the dance literature by providing a specific and replicable research protocol.

In meeting these outcomes, this study also makes important contributions to how social inclusion is operationalised and understood empirically. The qualitative research protocol involves the collection of rich descriptive data about participants' movements and interactions in the programme, as well as reflections on the dance experience immediately afterwards. As these observations and reflections are enhanced with interview and focus group data, the study will develop a better understanding of social inclusion as a process that unfolds over time and the programme/contextual factors that support it. ${ }^{28}$

Acknowledging that the majority of research on programmes and services for people living with dementia has relied disproportionately on the perspectives of carers, the development of this study design was purposefully collaborative with people living with dementia and with local Alzheimer Society client advisory committees and support groups, and will continue to include their perspectives in every phase of the study. Across the multiple phases of the study, research instruments are adapted based on feedback from people living with dementia involved in the research design and based on the sequential and iterative analysis of data. Such an approach will allow the continued development of more sensitive and inclusive research instruments and to improve the quality and impact of qualitative health research in the field. ${ }^{28}$

\section{DISCUSSION}

The qualitative research protocol outlined above adds to what is known already about the benefits of art-based health programmes, with its specific focus on social inclusion as a multidimensional process and outcome. The Improving Social Inclusion for People with Dementia and Carers through Sharing Dance study includes the perspectives of people living with dementia as well as community partners in a range of settings to understand better the contextual factors influencing the successes and failures of a dance programme for people living with dementia and carers. A strength of the study is the emphasis on data collection in institutional, community and household care settings in 
two comparable subnational (provincial) jurisdictions. A limitation is that this is a pilot study with an exploratory qualitative design and, as such, cannot provide quantitative outcome measures of the effectiveness of programme under investigation.

Producing new knowledge about the experiences, effectiveness and challenges of innovative arts-based programmes such as Baycrest NBS Sharing Dance Seniors, will inform the implementation of promising social inclusion programmes and, ultimately, has the potential to contribute to improving quality of life for older people living with dementia and their carers. The findings will inform the scalability of the programme, and provide evidence to support the development of other dance-based programmes and, more generally, policies regarding arts-based health programmes to improve social inclusion. The study presented herein also provides an example of the potential for community-based, qualitative health research to inform dementia care and ageing more generally.

Acknowledgements The authors are grateful for the contributions of knowledge users,collaborators and community partners from Alzheimer Society of Manitoba, Alzheimer Society of Ontario, Alzheimer Society of Peterborough, Kawartha Lakes, Northumberland and Haliburton, Baycrest Health Sciences, Canada's National Ballet School, Canada's Royal Winnipeg Ballet School, Canadian Consortium of Neurodegeneration in Aging, Community Care Peterborough, Minnedosa 50+ Activity Centre, Prairie Oasis Seniors Centre and Revera.

Contributors MWS and RVH are the study co-principal investigators and led the writing of the manuscript. PK and VM are the project co-investigators and RJB is the project research coordinator, who each contributed to writing and revising the manuscript.

Funding The Improving Social Inclusion for Canadians with Dementia and Carers through Sharing Dance study is funded by a Canadian Institutes of Health Research/ Alzheimer Society of Canada Operating Grant: Social Inclusion of Individuals with Dementia and Carers (CIHR/ASC grant no. 150702). The study is also funded, in part, by the Canada Research Chairs programme (Mark Skinner, Trent University; Rachel Herron, Brandon University).

Competing interests None declared.

Patient consent Not required.

Ethics approval This study received approval from the university research ethics boards at Trent University and Brandon University.

Provenance and peer review Not commissioned; peer reviewed for ethical and funding approval prior to submission.

Open access This is an open access article distributed in accordance with the Creative Commons Attribution Non Commercial (CC BY-NC 4.0) license, which permits others to distribute, remix, adapt, build upon this work non-commercially, and license their derivative works on different terms, provided the original work is properly cited, appropriate credit is given, any changes made indicated, and the use is non-commercial. See: http://creativecommons.org/licenses/by-nc/4.0/.

\section{REFERENCES}

1. International Alzheimer's Disease. The global impact of dementia: An analysis of prevalence, incidence, cost and trends. London: Alzheimer Disease International, 2015.

2. Clarke CL, Bailey C. Narrative citizenship, resilience and inclusion with dementia: On the inside or on the outside of physical and social places. Dementia 2016;15:434-52.
3. Roland KP, Chappell NL. Meaningful activity for persons with dementia: family caregiver perspectives. Am J Alzheimers Dis Other Demen 2015;30:559-68.

4. Walsh K, O'Shea E, Scharf T. Social Exclusion and Ageing in Diverse Rural Communities. National University of Ireland Galway: Irish Centre for Social Gerontology, 2012. www.icsg.ie/sites/www.icsg.ie/ files/personfiles/social_exclusion_and_rural_ageing.pdf.

5. Herron RV, Rosenberg MW. "Not there yet": Examining community support from the perspective of people with dementia and their partners in care. Soc Sci Med 2017;173:81-7.

6. Warburton J, Sh N, Shardlow SM. Social inclusion in an ageing world. Ageing \& Society 2013;33:1-15.

7. Kontos P, Miller KL, Mitchell GJ, et al. Presence redefined: The reciprocal nature of engagement between elder-clowns and persons with dementia. Dementia 2017;16:46-66.

8. Kontos P, Grigorovich A. Rethinking musicality in dementia as embodied and relational. J Aging Stud 2018;45:39-48.

9. Kontos P, Grigorovich A. Dancing with dementia: Citizenship, embodiment, and everyday life in long-term care. Katz S, ed. Ageing and everyday life: Materialities and embodiments. Bristol: Policy Press, 2018b:p.163-179.

10. Lapum JL, Bar RJ. Dance for Individuals With Dementia. J Psychosoc Nurs Ment Health Serv 2016;54:31-4.

11. Beard RL. Art therapies and dementia care: a systematic review. Dementia 2012;11:633-56.

12. Canada's National Ballet School (CNBS) website. Sharing Dance. 2018 www.nbs-enb.ca/Sharing-Dance.

13. Willis CD, Greene JK, Abramowicz A, et al. Strengthening the evidence and action on multi-sectoral partnerships in public health: an action research initiative. Health Promot Chronic Dis Prev Can 2016;36:101-11.

14. Guzmán $A$, Robinson $L$, Rochester $L$, et al. A process evaluation of a Psychomotor Dance Therapy Intervention (DANCIN) for behavior change in dementia: attitudes and beliefs of participating residents and staff. Int Psychogeriatr 2017;29:313-22.

15. Coubard O. Practice of contemporary dance improves cognitive flexibility in aging. Front Aging Neurosci 2011;3:1-12.

16. Abreu M, Hartley G. The effects of Salsa dance on balance, gait, and fall risk in a sedentary patient with Alzheimer's dementia, multiple comorbidities, and recurrent falls. J Geriatr Phys Ther 2013;36:100-8

17. Guzmán-García A, Hughes JC, James IA, et al. Dancing as a psychosocial intervention in care homes: a systematic review of the literature. Int J Geriatr Psychiatry 2013;28:914-24.

18. Young R, Camic PM, Tischler V. The impact of community-based arts and health interventions on cognition in people with dementia: a systematic literature review. Aging Ment Health 2016;20:337-51.

19. Dupuis SL, Kontos P, Mitchell G, et al. Re-claiming citizenship through the arts. Dementia 2016;15:358-80.

20. MacLeod A, Skinner MW, Wilkinson F, et al. Connecting socially isolated older rural adults with older volunteers through expressive arts. Can J Aging / La Revue canadienne du vieillissement 2016;35:14-27.

21. Peterborough O. Census Community Profile. Statistics Canada. Ottawa. 2016a, 2011. www.statcan.gc.ca.

22. Brandon M. Census Community Profile Statistics Canada. Ottawa. 2016b, 2011. www.statcan.gc.ca.

23. Keating N, Swindle J, Fletcher S. Aging in rural Canada: a retrospective and review. Can J Aging 2011;30:323-38.

24. Herron RV, Rosenberg MW, Skinner MW. The dynamics of voluntarism in rural dementia care. Health Place 2016;41:34-41.

25. Marshall F, Basiri A, Riley M, et al. Scaling the peaks research protocol: understanding the barriers and drivers to providing and using dementia-friendly community services in rural areas-a mixed methods study. BMJ Open 2018;8:e020374-10.

26. Mandelbaum R, Lo AC. Examining dance as an intervention in parkinson's disease: a systematic review. American Journal of Dance Therapy 2014;36:160-75.

27. Shanahan J, Morris ME, Bhriain ON, et al. Dance for people with parkinson disease: what is the evidence telling us? Arch Phys Med Rehabil 2015;96:141-53.

28. Herron R, Skinner M, Kontos P, et al. Beyond therapy: exploring the potential of dance to improve social inclusion for people with dementia. In: Hunt R, Atkinson S, eds. Geohumanities and Health, Springer, 2019. 